\title{
La competenza linguistica dei giovani italiani: cosa c'è al di là dei numeri?
}

Rosario Coluccia

PUBBLICATO: 03 SETTEMBRE 2019

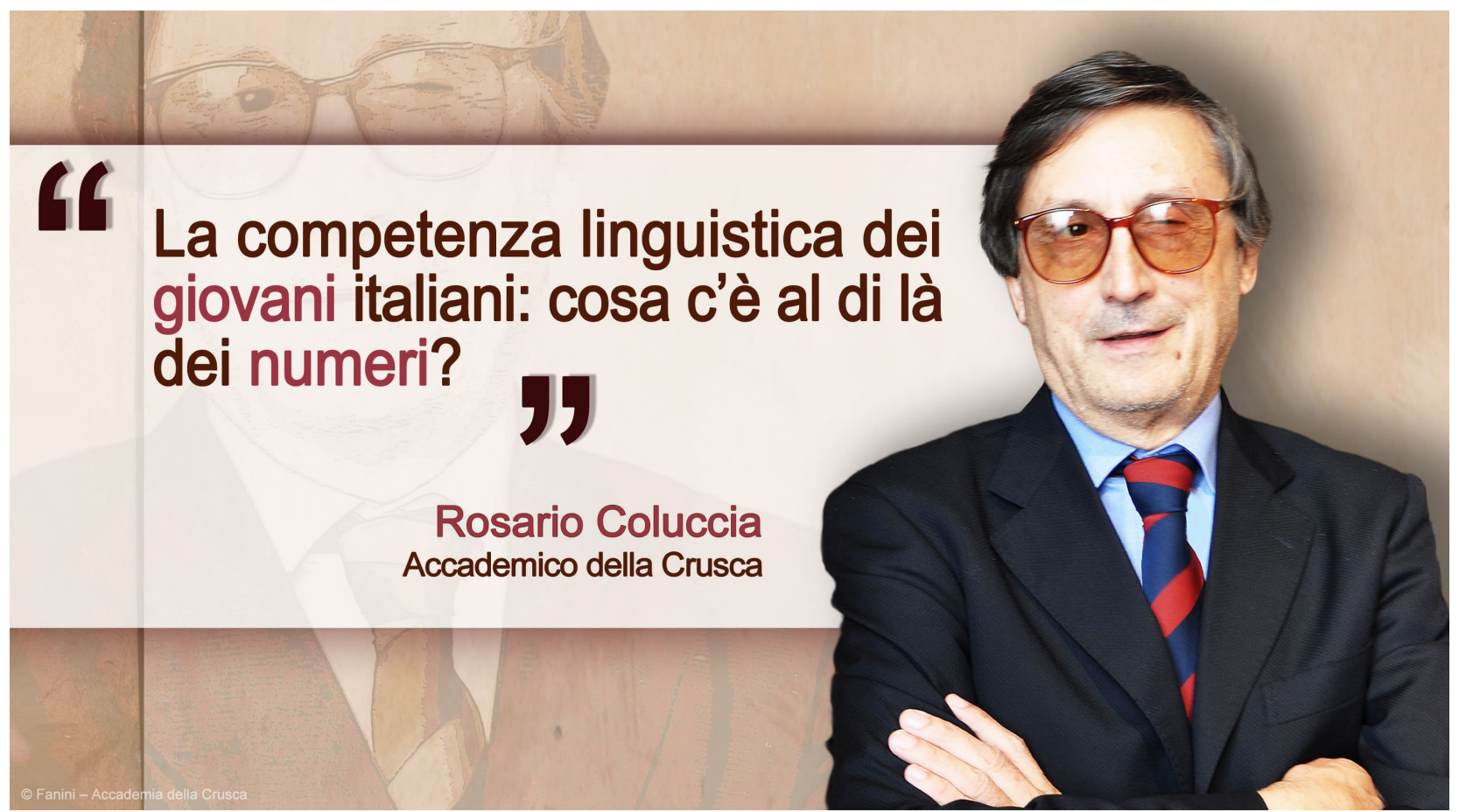

$\mathbf{L}$ uglio 20rg: si sono appena conclusi gli esami di maturità, il nuovo esame di Stato al debutto quest'anno. Si sono diplomati in Italia circa 520 mila studenti, con voti assai variabili, dal 60 (che è il minimo) al roo e lode (che è il massimo). Ovvio, i ragazzi non sono tutti ugualmente bravi. I risultati variano anche a seconda del tipo di scuola (liceo classico, scientifico, industriale, ecc.), dei contesti ambientali, della dislocazione regionale. Il confronto tra i punteggi di quest'anno e quelli dell'anno passato, segmentati per blocchi di voti (promossi con 6o; con punteggi compresi tra 6r e 70; tra 7r e 8o, tra 8r e 9o; ecc.) registra piccoli scostamenti in più o in meno, non rilevanti. Un paio di percentuali merita attenzione. I diplomati finali sono il 99,7\%, contro il 99,6\% dell'anno scorso. Inoltre, rispetto all'anno precedente, aumenta la percentuale di studenti che si maturano con il massimo dei voti: i diplomati con roo e lode sono l'ı,6\% (nei licei la cifra sale al 2,5\%), mentre l'anno scorso erano l'ı, $3 \%$. La percentuale dei promossi è altissima e aumenta anche il numero degli studenti che ottengono il punteggio massimo. Un sito commenta: "Da questi numeri si può capire che gli studenti italiani migliorano di anno in anno e che, in particolare, i maturandi che hanno appena affrontato la maturità sono stati davvero bravi rispetto ai colleghi degli scorsi anni".

Ma è davvero così? Altre verifiche danno risultati contraddittori. Da una decina d'anni nelle scuole italiane si svolgono le cosiddette prove Invalsi ("Istituto nazionale per la valutazione del sistema educativo di istruzione e di formazione") che intendono misurare le competenze in italiano, matematica e inglese degli studenti di elementari, medie e superiori. Non sono banali, vige un sistema di controllo piuttosto rigoroso. Dai test Invalsi risulta che alle superiori uno studente su tre non è in condizione di capire un testo in italiano di media complessità, senza contenuti tecnici o astrusità 
particolari. E dunque. Di fronte a una percentuale di promossi nelle scuole che rasenta il Ioo\%, in altre prove molti studenti ottengono risultati scarsi o scarsissimi, comunque insufficienti.

Si tratta di diversità dovute, semplicemente, a diversi criteri di analisi o (addirittura) a errori di valutazione commessi dai ricercatori? Per capirne di più, conviene allargare lo spettro dei confronti. PISA ("Programme for International Student Assessment") è una sigla che indica un'indagine dell'OCSE ("Organizzazione per la cooperazione e lo sviluppo economico") che valuta l'efficacia del sistema educativo mondiale. Vengono esaminati circa 540 mila studenti di 15 anni, rappresentativi di circa 30 milioni di coetanei, dislocati in 72 paesi, con tradizioni, storia, economie diverse. Si tratta di numeri molto alti, che non è possibile sottovalutare. La prima indagine PISA si è svolta nel 200o, viene ripetuta ogni tre anni (l'ultima è del 20I8), allo scopo di misurare anche le linee di tendenza, i miglioramenti e i peggioramenti.

Si considerano matematica, scienze e padronanza linguistica (naturalmente la lingua madre cambia paese per paese, nel nostro caso si considera la padronanza dell'italiano). Queste discipline sono alla base delle conoscenze e delle abilità necessarie per una piena partecipazione dell'individuo alla vita della società moderna, rispondono alla domanda: "Cosa è importante per un cittadino conoscere ed essere capace di fare"? Ai primi posti della più recente classifica troviamo Singapore, Giappone, Estonia, Taipei (/Formosa), Finlandia, Macao (Cina), Canada, Viet Nam, Hong Kong (Cina), Cina. Ce n'è abbastanza per smentire presupposizioni infondate (sull'eccellenza del sistema educativo occidentale tradizionale) e pregiudizi (sulle nazioni ritenute arretrate). L'Italia si colloca più o meno a metà classifica. Non c'è da consolarsi, siamo lontani dai vertici, battiamo paesi con economie povere e spesso dilaniati da guerre recenti; agli ultimi posti si collocano Tunisia, Macedonia, Kosovo, Algeria, Repubblica Domenicana.

Se si scompongono i dati l'allarme aumenta. Esistono differenze tra gli studenti italiani del Nord e quelli del Sud e delle Isole. Confrontati con la graduatoria globale, i primi (Bolzano, Trento e la Lombardia) raggiungono la media più alta, i secondi affondano in classifica nelle ultime posizioni. Gli studenti della Campania sono nella parte bassa, al pari dei ragazzi delle Azzorre e dell'Argentina. E allora converrà riconsiderare con estrema attenzione (non dico con sospetto) i risultati della maturità 2019, che indicano nella Campania la Regione con il piu alto numero assoluto di diplomati con lode (I.287), seguita da Puglia (I.225) e da Sicilia (8I7); se si considera invece il rapporto tra diplomati con lode e diplomati totali il risultato migliore viene raggiunto dalla Puglia (3.4\%), seguita dalla Calabria (2.6\%) e dall'Umbria (2.4\%) (la media nazionale è dell'ı,3\%).

Forse qualcosa non va nei voti alti concentrati in larga parte in alcune regioni. Né ha senso invocare un sorta di federalismo degli esami, assurdamente compensativo rispetto al federalismo di risorse, sanità, retribuzioni (anche dei docenti) che i governatori di Veneto e Lombardia reclamano per le loro regioni, in nome di un'autonomia mal intesa. Non è questo il punto cruciale. Al di là di qualche oscillazione dei voti, il problema di una scarsa conoscenza dell'italiano è generale, riguarda il paese intero. Alla fine del percorso scolastico troppi ragazzi scrivono male in italiano, leggono poco e faticano a esprimersi oralmente.

Fino a pochi decenni fa avevamo un'ottima scuola primaria, un liceo classico dove si studiava con profitto. Oggi le cose sono mutate. La scuola, lasciata sola a combattere i "mali del mondo" (dal riscaldamento globale alla ludopatia, dal ciberbullismo ai disturbi alimentari), si trova in difficoltà di fronte all'obiettivo di garantire una preparazione adeguata a tutti i frequentanti. Inutile lamentarsi dei risultati Invalsi se non si dotano le scuole di biblioteche, se non si fanno arrivare i libri nelle case in cui mancano, se non si investe in modo sistematico nella promozione della lettura. I dati sulla povertà 
dei ragazzi (economica ed educativa), sul numero dei lettori, sullabbandono scolastico, sulla disoccupazione giovanile narrano tutti la stessa storia: il fallimento dello Stato nel rimuovere gli ostacoli materiali e morali per la realizzazione di una piena uguaglianza.

Nel gennaio 2017 e nei mesi successivi fece molto rumore il cosiddetto appello dei seicento, sottoscritto da un folto gruppo di intellettuali, scrittori, giornalisti (non solo professori) che si intitolava "Saper leggere e scrivere: una proposta contro il declino dell'italiano a scuola". Se ne parlò molto, a quel tempo, anche con pareri diversi. Condivisa fu la constatazione che il dominio dellitaliano da parte dei giovani è in netto declino. Aggiungo: lo smottamento linguistico non coinvolge solo i giovani, è generalizzato. Errori grossolani pullulano sui media, né va meglio con professioni (magistratura, avvocatura) che pure hanno la lingua, il nostro più importante bene culturale immateriale, al centro della propria attività. L'uso maldestro dell'italiano, anche fuori dalla scuola e dall'università, è incontestabile. L'analfabetismo di ritorno è diffuso, molti adulti ne soffrono. E nulla di serio si propone (né, tanto meno, si fa) per contrastarlo.

Vengo al nodo, non tutti saranno d'accordo. Basta con le facilitazioni, comunque motivate. Bisogna puntare sulla qualità, a tutti i livelli. Invece si bada ai numeri, disinteressandosi dei contenuti, cedendo alle pressioni dell'ambiente e al sindacalismo dei genitori, che rivendicano a priori voti alti per i propri figli. Non cambia all'università. Ci sono corsi in cui molti si laureano in anticipo rispetto agli anni previsti e la media del voto di laurea oscilla tra iıo e iı e lode. Il Ministero premia questi corsi, ritenendo tali risultati eccellenti (e quindi incoraggia l'imitazione del modello); ma ho forti dubbi che quegli studenti siano tutti dei geni. Si premia l'apparenza, non la sostanza. Non rendiamo un buon servizio agli studenti se mettiamo sul medesimo pregevole piano chi studia duramente, con fatica, spesso in condizioni economiche disagiate, e chi ottiene gli stessi risultati immeritatamente. Ieri si guardava con ammirazione chi, a prezzo di sacrifici, riusciva a raggiungere livelli elevati di preparazione. Oggi invece prevale la convinzione che studio e sapere (privi di prestigio) sono irrilevanti per il successo sociale ed economico (ma non è così, un livello di competenze elevato dà anche vantaggi economici). La spinta generalizzata verso l'alto, indipendentemente dalle qualità e dallimpegno, non fa bene alla nostra scuola e alla nostra università.

La vera sfida è puntare sull'eccellenza reale, misurandosi alla pari con il resto d'Europa e con il mondo. Sono necessari investimenti strutturali: edifici, palestre, libri, attrezzature e strumenti didattici. Non sarebbe spesa improduttiva. I professori, già chiamati a un lavoro improbo e scarsamente considerato, abbiano voglia di porsi obiettivi ambiziosi, offrendo ulteriore impegno e chiedendo ai ragazzi qualità. Creare una vera unità nazionale nell'istruzione dovrebbe essere scopo primario della politica e obiettivo del paese intero. Servono giovani preparati, all'altezza dei tempi.

La partita si gioca nella scuola, li si vince o si perde tutto. Non ho ricette da proporre. Offro alla valutazione dei lettori solo modesti spunti di riflessione.

- Chi ha il potere di decidere non ceda alla velleità di varare ulteriori riforme scolastiche. Ogni governo tenta la propria, puntualmente smantellata dal governo successivo. Per qualche anno lasciamo che i professori, vessati dalla compilazione di questionari, di moduli e da adempimenti burocratici vari, possano lavorare tranquillamente, senza novità normative. La politica, senza clamore, si occupi di aumentare gli stipendi dei professori della scuola (oggi quasi indecorosi), di allestire biblioteche ricche di libri e laboratori ben attrezzati.

- I professori non rinunzino a esigere dagli studenti cognizioni, nozioni, date (un tempo giudicate essenziali e oggi ritenute inutili). Non è possibile l'apprendimento se mancano l'approfondimento e la riflessione individuali. Capire quello che si legge, parlare e scrivere 
correttamente richiedono applicazione e studio. Sono insensate le parole d'ordine che invitano i ragazzi a non studiare perché è inutile (essere competenti non serve!) e i professori a non dare compiti a casa (tutto deve concludersi in classe, senza fatica!). Ritorni nella scuola l'esercizio della memoria, facoltà importantissima alla quale abbiamo rinunziato a cuor leggero. Non sto esaltando il nozionismo, sto invitando alla conoscenza.

- Gli studenti, abituati alla comunicazione spezzettata dei social, allo scorrere frenetico di informazioni e di immagini, alla perpetua connessione in rete, si addestrino a distinguere, confrontare, scegliere nel mare di notizie complesse e contraddittorie disponibili fuori dalla scuola. Con la guida dei professori, cerchino nella scuola e nei libri di testo i percorsi per la loro preparazione.

- Le università preparino in modo adeguato i futuri insegnanti, che spesso posseggono in misura ancora limitata le nozioni di linguistica indispensabili per un efficace insegnamento dellitaliano. Dopo la laurea è necessario un aggiornamento costante degli insegnanti, di cui dovranno farsi carico ancora le università, insieme ad Accademie come la Crusca e i Lincei, ad Associazioni come l'ASLI e la SLI, che con grande merito già operano in quest'ambito. Università, Accademie e Associazioni facciano questo con le proprie risorse, senza spese per i partecipanti, sottraendo gli stessi al diluvio di corsi, corsetti e master di pessima qualità, organizzati da individui poco seri che dell'aggiornamento nelle scuole hanno fatto un mestiere lucroso.

Il tema riprende, con modifiche, due articoli apparsi in "Nuovo Quotidiano di Puglia", il 4 e il ı8 agosto 2019 .

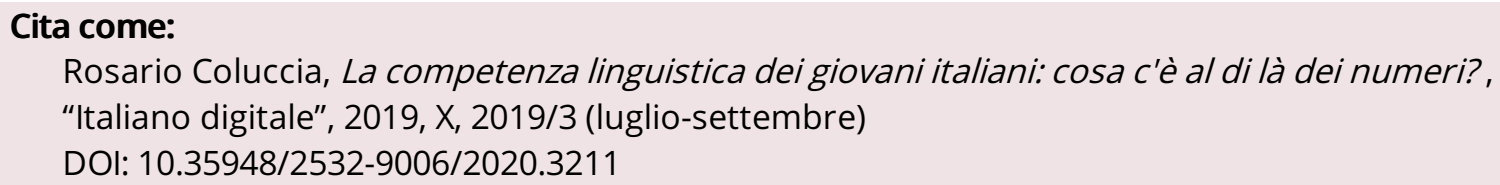

